DOI: $10.15393 /$ j3.art.2017.3790

UDC 517.51

S. S. DRAGOMIR

\title{
INEQUALITIES OF HERMITE-HADAMARD TYPE FOR HG-CONVEX FUNCTIONS
}

\begin{abstract}
Some inequalities of Hermite-Hadamard type for $H G$ convex functions defined on positive intervals are given. Applications for special means are also provided.
\end{abstract}

Key words: convex functions, integral inequalities, HG-convex functions

2010 Mathematical Subject Classification: 26D15, 25D10

1. Introduction. Following [4] (see also [26]) we say that the function $f: I \subset \mathbb{R} \backslash\{0\} \rightarrow \mathbb{R}$ is HA-convex if

$$
f\left(\frac{x y}{t x+(1-t) y}\right) \leq(1-t) f(x)+t f(y)
$$

for all $x, y \in I$ and $t \in[0,1]$. If the inequality in (1) is reversed, then $f$ is said to be $H A$-concave.

If $I \subset(0, \infty)$ and $f$ is convex and nondecreasing function then $f$ is $H A$-convex and if $f$ is $H A$-convex and nonincreasing function then $f$ is convex.

If $[a, b] \subset I \subset(0, \infty)$ and if we consider the function $g:\left[\frac{1}{b}, \frac{1}{a}\right] \rightarrow \mathbb{R}$, defined by $g(t)=f\left(\frac{1}{t}\right)$, then we can state the following fact [4]:

Lemma 1. The function $f$ is $H A$-convex on $[a, b]$ if and only if $g$ is convex in the usual sense on $\left[\frac{1}{b}, \frac{1}{a}\right]$.

Therefore, as examples of $H A$-convex functions we can take $f(t)=$ $=g\left(\frac{1}{t}\right)$, where $g$ is any convex function on $\left[\frac{1}{b}, \frac{1}{a}\right]$.

In the recent paper [16] we obtained the following characterization result as well: 
Lemma 2. Let $f, h:[a, b] \subset(0, \infty) \rightarrow \mathbb{R}$ be so that $h(t)=t f(t)$ for $t \in[a, b]$. Then $f$ is HA-convex on the interval $[a, b]$ if and only if $h$ is convex on $[a, b]$.

Following [4] (see also [26]) we say that the function $f: I \subset \mathbb{R} \backslash\{0\} \rightarrow$ $\rightarrow(0, \infty)$ is $H G$-convex if

$$
f\left(\frac{x y}{t x+(1-t) y}\right) \leq[f(x)]^{1-t}[f(y)]^{t}
$$

for all $x, y \in I$ and $t \in[0,1]$. If the inequality in $(2)$ is reversed, then $f$ is said to be $H G$-concave.

By the geometric-mean - arithmetic mean inequality we have that any $H G$-convex function is $H A$-convex. The converse is obviously not true.

We observe that $f: I \subset \mathbb{R} \backslash\{0\} \rightarrow(0, \infty)$ is $H G$-convex if and only if the function $\ln f: I \subset \mathbb{R} \backslash\{0\} \rightarrow \mathbb{R}$ is $H A$-convex on $I$.

Using Lemmas 1 and 2 we have:

Theorem 1. Let $f:[a, b] \subset(0, \infty) \rightarrow(0, \infty)$ and define the associated functions $G_{f}:\left[\frac{1}{b}, \frac{1}{a}\right] \rightarrow \mathbb{R}$ defined by $G_{f}(t)=\ln f\left(\frac{1}{t}\right)$ and $H_{f}:[a, b] \subset$ $\subset(0, \infty) \rightarrow \mathbb{R}$ defined by $H_{f}(t)=t \ln f(t)$. Then the following statements are equivalent:

(i) The function $f$ is $H G$-convex on $[a, b]$;

(ii) The function $G_{f}$ is convex on $\left[\frac{1}{b}, \frac{1}{a}\right]$;

(iii) The function $H_{f}$ is convex on $[a, b]$.

For a convex function $h:[c, d] \rightarrow \mathbb{R}$, the following inequality is well known in the literature as the Hermite-Hadamard inequality

$$
h\left(\frac{c+d}{2}\right) \leq \frac{1}{d-c} \int_{c}^{d} h(t) d t \leq \frac{h(c)+h(d)}{2} .
$$

For related results, see [1]- 10, [12]- 28].

Motivated by the above results, we establish in this paper some inequalities of Hermite-Hadamard type for $H G$-convex functions defined on positive intervals. Applications for special means are also provided.

2. Main Results. The following result holds.

Theorem 2. Let $f:[a, b] \subset(0, \infty) \rightarrow(0, \infty)$ be an HG-convex function on the interval $[a, b]$. Then for any $\lambda \in[0,1]$ we have the inequalities

$$
f\left(\frac{2 a b}{a+b}\right) \leq\left[f\left(\frac{2 a b}{(1-\lambda) a+(\lambda+1) b}\right)\right]^{1-\lambda}\left[f\left(\frac{2 a b}{(2-\lambda) a+\lambda b}\right)\right]^{\lambda} \leq
$$




$$
\begin{gathered}
\leq \exp \left(\frac{a b}{b-a} \int_{a}^{b} \frac{\ln f(t)}{t^{2}} d t\right) \leq \\
\leq \sqrt{f\left(\frac{a b}{(1-\lambda) a+\lambda b}\right)[f(a)]^{1-\lambda}[f(b)]^{\lambda}} \leq \sqrt{f(a) f(b)} .
\end{gathered}
$$

If we take $\lambda=\frac{1}{2}$ in 4 , then we get

$$
\begin{gathered}
f\left(\frac{2 a b}{a+b}\right) \leq \sqrt{f\left(\frac{4 a b}{a+3 b}\right) f\left(\frac{4 a b}{3 a+b}\right)} \leq \\
\leq \exp \left(\frac{a b}{b-a} \int_{a}^{b} \frac{\ln f(t)}{t^{2}} d t\right) \leq \\
\leq \sqrt{f\left(\frac{2 a b}{a+b}\right) \sqrt{f(a) f(b)}} \leq \sqrt{f(a) f(b)} .
\end{gathered}
$$

The identric mean $I(a, b)$ for two distinct positive numbers $a, b$ is defined by

$$
I(a, b):=\frac{1}{e}\left(\frac{b^{b}}{a^{a}}\right)^{\frac{1}{b-a}}
$$

while the logarithmic mean is defined by

$$
L(a, b):=\frac{b-a}{\ln b-\ln a} .
$$

Theorem 3. Let $f:[a, b] \subset(0, \infty) \rightarrow(0, \infty)$ be an HG-convex function on the interval $[a, b]$. Then

$f(L(a, b)) \leq \exp \left(\frac{1}{b-a} \int_{a}^{b} \ln f(t) d t\right) \leq[f(b)]^{\frac{(L(a, b)-a) b}{(b-a) L(a, b)}}[f(a)]^{\frac{(b-L(a, b)) a}{(b-a) L(a, b)}}$.

If we write the classical Hermite-Hadamard inequality for the function $H_{f}$ that is convex on $[a, b]$ when $f:[a, b] \subset(0, \infty) \rightarrow(0, \infty)$ is an $H G$ convex function on $[a, b]$ and perform the required calculations, we get: 
Theorem 4. Let $f:[a, b] \subset(0, \infty) \rightarrow(0, \infty)$ be an HG-convex function on the interval $[a, b]$. Then we have

$$
\left[f\left(\frac{a+b}{2}\right)\right]^{\frac{a+b}{2}} \leq \exp \left(\frac{1}{b-a} \int_{a}^{b} t \ln f(t) d t\right) \leq \sqrt{[f(b)]^{b}[f(a)]^{a}} .
$$

We have the reverse inequalities as well:

Theorem 5. Let $f:[a, b] \subset(0, \infty) \rightarrow(0, \infty)$ be an HG-convex function on the interval $[a, b]$. Then we have

$$
\begin{gathered}
1 \leq \frac{\exp \left(\frac{a b}{b-a} \int_{a}^{b} \frac{\ln f(t)}{t^{2}} d t\right)}{f\left(\frac{2 a b}{a+b}\right)} \leq \\
\leq \exp \left(\frac{1}{8}\left[\frac{f_{-}^{\prime}(b)}{f(b)} b^{2}-\frac{f_{+}^{\prime}(a) a^{2}}{f(a)}\right]\left(\frac{b-a}{a b}\right)\right)
\end{gathered}
$$

and

$$
\begin{gathered}
1 \leq \frac{\sqrt{f(a) f(b)}}{\exp \left(\frac{a b}{b-a} \int_{a}^{b} \frac{\ln f(t)}{t^{2}} d t\right)} \leq \\
\leq \exp \left(\frac{1}{8}\left[\frac{f_{-}^{\prime}(b)}{f(b)} b^{2}-\frac{f_{+}^{\prime}(a) a^{2}}{f(a)}\right]\left(\frac{b-a}{a b}\right)\right) .
\end{gathered}
$$

The following related result also holds:

Theorem 6. Let $f:[a, b] \subset(0, \infty) \rightarrow(0, \infty)$ be an HG-convex function on the interval $[a, b]$. Then we have

$$
\begin{gathered}
1 \leq \frac{\sqrt{[f(a)]^{a}[f(b)]^{b}}}{\exp \left(\frac{1}{b-a} \int_{a}^{b} t \ln f(t) d t\right)} \leq \\
\leq\left(\frac{f(b)}{f(a)}\right)^{\frac{1}{8}(b-a)} \exp \left(\frac{1}{8}(b-a)\left(\frac{b f_{-}^{\prime}(b)}{f(b)}-\frac{a f_{+}^{\prime}(a)}{f(a)}\right)\right)
\end{gathered}
$$


and

$$
\begin{gathered}
1 \leq \frac{\exp \left(\frac{1}{b-a} \int_{a}^{b} t \ln f(t) d t\right)}{\left[f\left(\frac{a+b}{2}\right)\right]^{\frac{a+b}{2}}} \leq \\
\leq\left(\frac{f(b)}{f(a)}\right)^{\frac{1}{8}(b-a)} \exp \left(\frac{1}{8}(b-a)\left(\frac{b f_{-}^{\prime}(b)}{f(b)}-\frac{a f_{+}^{\prime}(a)}{f(a)}\right)\right) .
\end{gathered}
$$

From a different perspective we have:

Theorem 7. Let $f:[a, b] \subset(0, \infty) \rightarrow(0, \infty)$ be an $H G$-convex function on the interval $[a, b]$. Then

$$
\exp \left(\frac{a b}{b-a} \int_{a}^{b} \frac{\ln f(t)}{t^{2}} d t\right) \leq \sqrt{f(x)[f(b)]^{\frac{a(b-x)}{x(b-a)}}[f(a)]^{\frac{b(x-a)}{x(b-a)}}}
$$

for any $x \in[a, b]$.

If we take in $12, x=\frac{a+b}{2}$, then we get from 12 that

$$
\exp \left(\frac{a b}{b-a} \int_{a}^{b} \frac{\ln f(t)}{t^{2}} d t\right) \leq \sqrt{f\left(\frac{a+b}{2}\right)[f(b)]^{\frac{a}{a+b}}[f(a)]^{\frac{b}{a+b}}} .
$$

3. Proofs. In [15, in order to improve Işcan's inequality [26] for $H A$-convex functions $g:[a, b] \subset(0, \infty) \rightarrow \mathbb{R}$,

$$
g\left(\frac{2 a b}{a+b}\right) \leq \frac{a b}{b-a} \int_{a}^{b} \frac{g(t)}{t^{2}} d t \leq \frac{g(a)+g(b)}{2},
$$

we obtained the following result:

$$
\begin{gathered}
g\left(\frac{2 a b}{a+b}\right) \leq(1-\lambda) g\left(\frac{2 a b}{(1-\lambda) a+(\lambda+1) b}\right)+ \\
+\lambda g\left(\frac{2 a b}{(2-\lambda) a+\lambda b}\right) \leq \frac{a b}{b-a} \int_{a}^{b} \frac{g(t)}{t^{2}} d t \leq
\end{gathered}
$$




$$
\begin{gathered}
\leq \frac{1}{2}\left[g\left(\frac{a b}{(1-\lambda) a+\lambda b}\right)+(1-\lambda) g(a)+\lambda g(b)\right] \leq \\
\leq \frac{g(a)+g(b)}{2},
\end{gathered}
$$

where $\lambda \in[0,1]$.

Now, if $f:[a, b] \subset(0, \infty) \rightarrow(0, \infty)$ is an $H G$-convex function on the interval $[a, b]$, then $g:=\ln f$ is $H A$-convex on $[a, b]$, and by 15 we get

$$
\begin{gathered}
\ln f\left(\frac{2 a b}{a+b}\right) \leq(1-\lambda) \ln f\left(\frac{2 a b}{(1-\lambda) a+(\lambda+1) b}\right)+ \\
+\lambda \ln f\left(\frac{2 a b}{(2-\lambda) a+\lambda b}\right) \leq \frac{a b}{b-a} \int_{a}^{b} \frac{\ln f(t)}{t^{2}} d t \leq \\
\leq \frac{1}{2}\left[\ln f\left(\frac{a b}{(1-\lambda) a+\lambda b}\right)+(1-\lambda) \ln f(a)+\lambda \ln f(b)\right] \leq \\
\leq \frac{\ln f(a)+\ln f(b)}{2},
\end{gathered}
$$

that is equivalent to

$$
\begin{gathered}
\ln f\left(\frac{2 a b}{a+b}\right) \leq \\
\leq \ln \left(\left[f\left(\frac{2 a b}{(1-\lambda) a+(\lambda+1) b}\right)\right]^{1-\lambda}\left[f\left(\frac{2 a b}{(2-\lambda) a+\lambda b}\right)\right]^{\lambda}\right) \leq \\
\leq \frac{a b}{b-a} \int_{a}^{b} \frac{\ln f(t)}{t^{2}} d t \leq \ln \sqrt{f\left(\frac{a b}{(1-\lambda) a+\lambda b}\right)[f(a)]^{1-\lambda}[f(b)]^{\lambda}} \leq \\
\leq \ln \sqrt{f(a) f(b)},
\end{gathered}
$$

and by taking the exponential we get the desired result (4).

We have the following result for $H A$-convex functions [15]:

Lemma 3. Let $g:[a, b] \subset(0, \infty) \rightarrow \mathbb{R}$ be an HA-convex function on the interval $[a, b]$. Then

$g(L(a, b)) \leq \frac{1}{b-a} \int_{a}^{b} g(x) d x \leq \frac{(L(a, b)-a) b g(b)+(b-L(a, b)) a g(a)}{(b-a) L(a, b)}$. 
If $f:[a, b] \subset(0, \infty) \rightarrow(0, \infty)$ is an $H G$-convex function on the interval $[a, b]$, then $g:=\ln f$ is $H A$-convex on $[a, b]$, and by (17) we have

$$
\begin{gathered}
\ln f(L(a, b)) \leq \frac{1}{b-a} \int_{a}^{b} \ln f(x) d x \leq \\
\leq \frac{(L(a, b)-a) b \ln f(b)+(b-L(a, b)) a \ln f(a)}{(b-a) L(a, b)}= \\
=\ln \left([f(b)]^{\frac{(L(a, b)-a) b}{(b-a) L(a, b)}}[f(a)]^{\frac{(b-L(a, b)) a}{(b-a) L(a, b)}}\right) .
\end{gathered}
$$

By taking the exponential in (18) we get the desired result (6).

We use the following results obtained by the author in [10] and [11:

Lemma 4. Let $h:[\alpha, \beta] \rightarrow \mathbb{R}$ be a convex function on $[\alpha, \beta]$. Then we have the inequalities

$$
0 \leq \frac{h(\alpha)+h(\beta)}{2}-\frac{1}{\beta-\alpha} \int_{\alpha}^{\beta} h(t) d t \leq \frac{1}{8}\left[h_{-}^{\prime}(\beta)-h_{+}^{\prime}(\alpha)\right](\beta-\alpha)
$$

and

$$
0 \leq \frac{1}{\beta-\alpha} \int_{\alpha}^{\beta} h(t) d t-h\left(\frac{\alpha+\beta}{2}\right) \leq \frac{1}{8}\left[h_{-}^{\prime}(\beta)-h_{+}^{\prime}(\alpha)\right](\beta-\alpha) .
$$

The constant $\frac{1}{8}$ is best possible in (19) and 20).

If $\ell:[a, b] \subset(0, \infty) \rightarrow \mathbb{R}$ is an $H A$-convex function on the interval $[a, b]$, then the function $g:\left[\frac{1}{b}, \frac{1}{a}\right] \rightarrow \mathbb{R}, g(s)=\ell\left(\frac{1}{s}\right)$, is convex on $\left[\frac{1}{b}, \frac{1}{a}\right]$.

Now, by (19) and (20) we have

$$
\begin{aligned}
0 & \leq \frac{g\left(\frac{1}{a}\right)+g\left(\frac{1}{b}\right)}{2}-\frac{1}{\frac{1}{a}-\frac{1}{b}} \int_{\frac{1}{b}}^{\frac{1}{a}} g(t) d t \leq \\
& \leq \frac{1}{8}\left[g_{-}^{\prime}\left(\frac{1}{a}\right)-g_{+}^{\prime}\left(\frac{1}{b}\right)\right]\left(\frac{1}{a}-\frac{1}{b}\right)
\end{aligned}
$$


and

$$
\begin{aligned}
& 0 \leq \frac{1}{\frac{1}{a}-\frac{1}{b}} \int_{\frac{1}{b}}^{\frac{1}{a}} g(t) d t-g\left(\frac{\frac{1}{a}+\frac{1}{b}}{2}\right) \leq \\
& \leq \frac{1}{8}\left[g_{-}^{\prime}\left(\frac{1}{a}\right)-g_{+}^{\prime}\left(\frac{1}{b}\right)\right]\left(\frac{1}{a}-\frac{1}{b}\right) .
\end{aligned}
$$

We also have

$$
g_{ \pm}^{\prime}(s)=\ell_{\mp}^{\prime}\left(\frac{1}{s}\right)\left(-\frac{1}{s^{2}}\right)
$$

and then

$$
g_{-}^{\prime}\left(\frac{1}{a}\right)=-\ell_{+}^{\prime}(a) a^{2} \text { and } g_{+}^{\prime}\left(\frac{1}{b}\right)=-\ell_{-}^{\prime}(b) b^{2} .
$$

From 21] and 22 we have

$$
\begin{aligned}
0 & \leq \frac{\ell(a)+\ell(b)}{2}-\frac{a b}{b-a} \int_{\frac{1}{b}}^{\frac{1}{a}} \ell\left(\frac{1}{s}\right) d s \leq \\
& \leq \frac{1}{8}\left[\ell_{-}^{\prime}(b) b^{2}-\ell_{+}^{\prime}(a) a^{2}\right]\left(\frac{b-a}{a b}\right)
\end{aligned}
$$

and

$$
\begin{aligned}
0 & \leq \frac{a b}{b-a} \int_{\frac{1}{b}}^{\frac{1}{a}} \ell\left(\frac{1}{s}\right) d s-\ell\left(\frac{2 a b}{a+b}\right) \leq \\
& \leq \frac{1}{8}\left[\ell_{-}^{\prime}(b) b^{2}-\ell_{+}^{\prime}(a) a^{2}\right]\left(\frac{b-a}{a b}\right) .
\end{aligned}
$$

If we change the variable $\frac{1}{s}=u$, then $d s=-\frac{d u}{u^{2}}$ and 23 and 24 can be written as

$$
\begin{aligned}
0 & \leq \frac{\ell(a)+\ell(b)}{2}-\frac{a b}{b-a} \int_{a}^{b} \frac{\ell(t)}{t^{2}} d t \leq \\
& \leq \frac{1}{8}\left[\ell_{-}^{\prime}(b) b^{2}-\ell_{+}^{\prime}(a) a^{2}\right]\left(\frac{b-a}{a b}\right)
\end{aligned}
$$


and

$$
\begin{aligned}
& 0 \leq \frac{a b}{b-a} \int_{a}^{b} \frac{\ell(t)}{t^{2}} d t-\ell\left(\frac{2 a b}{a+b}\right) \leq \\
& \leq \frac{1}{8}\left[\ell_{-}^{\prime}(b) b^{2}-\ell_{+}^{\prime}(a) a^{2}\right]\left(\frac{b-a}{a b}\right) .
\end{aligned}
$$

If $f:[a, b] \subset(0, \infty) \rightarrow(0, \infty)$ is an $H G$-convex function on the interval $[a, b]$, then $\ell:=\ln f$ is $H A$-convex on $[a, b]$, and by (25) and 26) we have

$$
\begin{aligned}
0 & \leq \ln \sqrt{f(a) f(b)}-\frac{a b}{b-a} \int_{a}^{b} \frac{\ln f(t)}{t^{2}} d t \leq \\
& \leq \frac{1}{8}\left[\frac{f_{-}^{\prime}(b)}{f(b)} b^{2}-\frac{f_{+}^{\prime}(a) a^{2}}{f(a)}\right]\left(\frac{b-a}{a b}\right)
\end{aligned}
$$

and

$$
\begin{aligned}
0 & \leq \frac{a b}{b-a} \int_{a}^{b} \frac{\ln f(t)}{t^{2}} d t-\ln f\left(\frac{2 a b}{a+b}\right) \leq \\
& \leq \frac{1}{8}\left[\frac{f_{-}^{\prime}(b)}{f(b)} b^{2}-\frac{f_{+}^{\prime}(a) a^{2}}{f(a)}\right]\left(\frac{b-a}{a b}\right),
\end{aligned}
$$

and the Theorem 5 is proved.

If $f:[a, b] \subset(0, \infty) \rightarrow(0, \infty)$ is an $H G$-convex function on the interval $[a, b]$, then $H_{f}$ is convex on $[a, b]$ and by 19$]$ and $(20)$ we have after appropriate calculations

$$
\begin{aligned}
& 0 \leq \ln \sqrt{[f(a)]^{a}[f(b)]^{b}}-\frac{1}{b-a} \int_{a}^{b} t \ln f(t) d t \leq \\
\leq & \frac{1}{8}\left[\ln f(b)+\frac{b f_{-}^{\prime}(b)}{f(b)}-\ln f(a)-\frac{a f_{+}^{\prime}(a)}{f(a)}\right](b-a)= \\
= & \ln \left(\frac{f(b)}{f(a)}\right)^{\frac{1}{8}(b-a)}+\frac{1}{8}(b-a)\left(\frac{b f_{-}^{\prime}(b)}{f(b)}-\frac{a f_{+}^{\prime}(a)}{f(a)}\right)
\end{aligned}
$$


and

$$
\begin{aligned}
& 0 \leq \frac{1}{b-a} \int_{a}^{b} t \ln f(t) d t-\ln \left(\left[f\left(\frac{a+b}{2}\right)\right]^{\frac{a+b}{2}}\right) \leq \\
& \leq \ln \left(\frac{f(b)}{f(a)}\right)^{\frac{1}{8}(b-a)}+\frac{1}{8}(b-a)\left(\frac{b f_{-}^{\prime}(b)}{f(b)}-\frac{a f_{+}^{\prime}(a)}{f(a)}\right) .
\end{aligned}
$$

These inequalities are equivalent to

$$
\begin{gathered}
0 \leq \ln \left(\frac{\sqrt{[f(a)]^{a}[f(b)]^{b}}}{\exp \left(\frac{1}{b-a} \int_{a}^{b} t \ln f(t) d t\right)}\right) \leq \\
\leq \ln \left[\left(\frac{f(b)}{f(a)}\right)^{\frac{1}{8}(b-a)} \exp \left(\frac{1}{8}(b-a)\left(\frac{b f_{-}^{\prime}(b)}{f(b)}-\frac{a f_{+}^{\prime}(a)}{f(a)}\right)\right)\right]
\end{gathered}
$$

and

$$
\begin{gathered}
0 \leq \ln \left(\frac{\exp \left(\frac{1}{b-a} \int_{a}^{b} t \ln f(t) d t\right)}{\left[f\left(\frac{a+b}{2}\right)\right]^{\frac{a+b}{2}}}\right) \leq \\
\leq \ln \left[\left(\frac{f(b)}{f(a)}\right)^{\frac{1}{8}(b-a)} \exp \left(\frac{1}{8}(b-a)\left(\frac{b f_{-}^{\prime}(b)}{f(b)}-\frac{a f_{+}^{\prime}(a)}{f(a)}\right)\right)\right]
\end{gathered}
$$

and by taking the exponential we get the desired results $(10)$ and $(11)$. The following lemma is of interest in itself:

Lemma 5. Let $g:[a, b] \subset(0, \infty) \rightarrow \mathbb{R}$ be a HA-convex function on the interval $[a, b]$. Then

$$
\frac{1}{2 x}\left(\frac{g(b) a(b-x)+g(a) b(x-a)}{b-a}+x g(x)\right) \geq \frac{a b}{b-a} \int_{a}^{b} \frac{g(y)}{y^{2}} d y
$$

for any $x \in[a, b]$. 
Proof. Since $h(t)=t g(t)$ for $t \in[a, b]$ is convex, then by the gradient inequality for convex functions we have

$$
x g(x)-y g(y) \geq\left(g(y)+y g_{-}^{\prime}(y)\right)(x-y)
$$

for any $x, y \in(a, b)$.

This is equivalent to

$$
x g(x)-x g(y) \geq y g_{-}^{\prime}(y)(x-y)
$$

for any $x, y \in(a, b)$.

From 30 we have, by division with $x y^{2}>0$, that

$$
\frac{1}{y^{2}} g(x)-\frac{1}{y^{2}} g(y) \geq \frac{g_{-}^{\prime}(y)}{y}\left(1-\frac{y}{x}\right)
$$

for any $x, y \in(a, b)$.

Taking the integral mean over $y$ we have

$$
\begin{aligned}
& g(x) \frac{1}{b-a} \int_{a}^{b} \frac{1}{y^{2}} d y-\frac{1}{b-a} \int_{a}^{b} \frac{g(y)}{y^{2}} d y \geq \\
& \geq \frac{1}{b-a} \int_{a}^{b} \frac{g_{-}^{\prime}(y)}{y} d y-\frac{1}{x} \frac{1}{b-a} \int_{a}^{b} g_{-}^{\prime}(y) d y
\end{aligned}
$$

that is equivalent to

$$
\begin{gathered}
\frac{g(x)}{a b}-\frac{1}{b-a} \int_{a}^{b} \frac{g(y)}{y^{2}} d y \geq \\
\geq \frac{1}{b-a}\left[\frac{g(b)}{b}-\frac{g(a)}{a}+\int_{a}^{b} \frac{g(y)}{y^{2}} d y\right]-\frac{1}{x} \frac{g(b)-g(a)}{b-a}= \\
=\frac{1}{b-a}\left(\frac{g(b)}{b}-\frac{g(a)}{a}\right)+\frac{1}{b-a} \int_{a}^{b} \frac{g(y)}{y^{2}} d y-\frac{1}{x} \frac{g(b)-g(a)}{b-a},
\end{gathered}
$$


for any $x \in(a, b)$. This can be written as

$$
\frac{1}{x} \frac{g(b)-g(a)}{b-a}-\frac{1}{b-a}\left(\frac{g(b)}{b}-\frac{g(a)}{a}\right) \geq \frac{2}{b-a} \int_{a}^{b} \frac{g(y)}{y^{2}} d y-\frac{g(x)}{a b}
$$

or as

$$
\frac{1}{2}\left(\frac{1}{b-a}\left[g(b) \frac{b-x}{x b}+g(a) \frac{x-a}{a x}\right]+\frac{g(x)}{a b}\right) \geq \frac{1}{b-a} \int_{a}^{b} \frac{g(y)}{y^{2}} d y .
$$

This is equivalent to the desired result 29.

If $f:[a, b] \subset(0, \infty) \rightarrow(0, \infty)$ is an $H G$-convex function on the interval $[a, b]$, then $g:=\ln f$ is $H A$-convex on $[a, b]$, and by 29$]$ we have

$$
\begin{gathered}
\frac{1}{2 x}\left(\frac{a(b-x) \ln f(b)+b(x-a) \ln f(a)}{b-a}+x \ln f(x)\right) \geq \\
\geq \frac{a b}{b-a} \int_{a}^{b} \frac{\ln f(y)}{y^{2}} d y
\end{gathered}
$$

for any $x \in[a, b]$.

This is clearly equivalent to

$$
\ln \left(\sqrt{[f(b)]^{\frac{a(b-x)}{x(b-a)}}[f(a)]^{\frac{b(x-a)}{x(b-a)}}} \sqrt{f(x)}\right) \geq \frac{a b}{b-a} \int_{a}^{b} \frac{\ln f(y)}{y^{2}} d y
$$

for any $x \in[a, b]$.

If we take the exponential in (31), then we get the desired result (12).

4. Applications. Consider the function $f:[a, b] \subset(0, \infty) \rightarrow(0, \infty)$, $f(t)=t$. Using the geometric mean - harmonic mean inequality, we have

$$
f\left(\frac{x y}{t x+(1-t) y}\right)=\frac{x y}{t x+(1-t) y} \leq x^{1-t} y^{t}=[f(x)]^{1-t}[f(y)]^{t},
$$

which shows that $f$ is $H G$-convex on $[a, b]$.

We need the following integrals

$$
\frac{1}{b-a} \int_{a}^{b} \ln f(t) d t=\frac{1}{b-a} \int_{a}^{b} \ln t d t=\ln I(a, b),
$$




$$
\begin{gathered}
\frac{1}{b-a} \int_{a}^{b} t \ln f(t) d t=\frac{1}{b-a} \int_{a}^{b} t \ln t d t= \\
=\frac{1}{2} A(a, b) \ln I\left(a^{2}, b^{2}\right)=\ln \left[I\left(a^{2}, b^{2}\right)^{\frac{1}{2} A(a, b)}\right]
\end{gathered}
$$

and

$$
\int_{a}^{b} \frac{\ln f(t)}{t^{2}} d t=\int_{a}^{b} \frac{\ln t}{t^{2}} d t=\frac{b-a}{a b} \ln \left[I\left(a^{-1}, b^{-1}\right)\right]^{-1}
$$

giving that

$$
\frac{a b}{b-a} \int_{a}^{b} \frac{\ln f(t)}{t^{2}} d t=\ln \left[I\left(a^{-1}, b^{-1}\right)\right]^{-1} .
$$

Now, if we write the inequality (4) for the function $f:[a, b] \subset(0, \infty) \rightarrow$ $\rightarrow(0, \infty), f(t)=t$, we get

$$
\begin{aligned}
& H(a, b) \leq\left(\frac{2 a b}{(1-\lambda) a+(\lambda+1) b}\right)^{1-\lambda}\left(\frac{2 a b}{(2-\lambda) a+\lambda b}\right)^{\lambda} \leq \\
\leq & {\left[I\left(a^{-1}, b^{-1}\right)\right]^{-1} \leq \sqrt{\left(\frac{a b}{(1-\lambda) a+\lambda b}\right) a^{1-\lambda} b^{\lambda}} \leq G(a, b), }
\end{aligned}
$$

where $H(a, b):=\frac{2 a b}{a+b}$ is the harmonic mean.

If we use the inequality (6) for $f(t)=t$, then we have

$$
(L(a, b) \leq) I(a, b) \leq b^{\frac{(L(a, b)-a) b}{(b-a) L(a, b)}} a^{\frac{(b-L(a, b)) a}{(b-a) L(a, b)}} .
$$

If we use the inequality (7) for $f(t)=t, t \in[a, b]$, then we also get

$$
[A(a, b)]^{A(a, b)} \leq I\left(a^{2}, b^{2}\right)^{\frac{1}{2} A(a, b)} \leq G\left(b^{b}, a^{a}\right) .
$$

From (8) and (9) for $f(t)=t$ we have

$$
1 \leq \frac{\left[I\left(a^{-1}, b^{-1}\right)\right]^{-1}}{H(a, b)} \leq \exp \left(\frac{(b-a)^{2}}{8 a b}\right)
$$

and

$$
1 \leq \frac{G(a, b)}{\left[I\left(a^{-1}, b^{-1}\right)\right]^{-1}} \leq \exp \left(\frac{(b-a)^{2}}{8 a b}\right)
$$


From $(10)$ and $(11)$ we also have

$$
1 \leq \frac{G\left(a^{a}, b^{b}\right)}{\left[I\left(a^{2}, b^{2}\right)\right]^{\frac{1}{2} A(a, b)}} \leq\left(\frac{b}{a}\right)^{\frac{1}{8}(b-a)}
$$

and

$$
1 \leq \frac{\left[I\left(a^{2}, b^{2}\right)\right]^{\frac{1}{2} A(a, b)}}{[A(a, b)]^{A(a, b)}} \leq\left(\frac{b}{a}\right)^{\frac{1}{8}(b-a)} .
$$

Finally, from 13 we obtain

$$
\left[I\left(a^{-1}, b^{-1}\right)\right]^{-1} \leq \sqrt{A(a, b) b^{\frac{a}{a+b}} a^{\frac{b}{a+b}}}
$$

Now consider the function $f:[a, b] \subset(0, \infty) \rightarrow(0, \infty), f(t)=\exp (t)$. Using the harmonic mean-arithmetic mean inequality we have

$$
\begin{gathered}
f\left(\frac{x y}{t x+(1-t) y}\right)=\exp \left(\frac{x y}{t x+(1-t) y}\right) \leq \exp ((1-t) x+t y)= \\
=[\exp (x)]^{1-t}[\exp (y)]^{t}=[f(x)]^{1-t}[f(y)]^{t}
\end{gathered}
$$

for any $x, y \in[a, b]$ and $t \in[0,1]$.

Now, if we use the inequality (4) for the $H G$-convex function $f:[a, b] \subset$ $\subset(0, \infty) \rightarrow(0, \infty), f(t)=\exp (t)$, then we get, after suitable calculations, that

$$
\begin{gathered}
H(a, b) \leq \frac{2(1-\lambda) a b}{(1-\lambda) a+(\lambda+1) b}+\frac{2 \lambda a b}{(2-\lambda) a+\lambda b} \leq \\
\leq \frac{G^{2}(a, b)}{L(a, b)} \leq \frac{1}{2}\left(\frac{a b}{(1-\lambda) a+\lambda b}+(1-\lambda) a+\lambda b\right) \leq A(a, b),
\end{gathered}
$$

for any $\lambda \in[0,1]$.

If we use the inequalities $(8)$ and $(9)$ for the $H G$-convex function $f$ : $[a, b] \subset(0, \infty) \rightarrow(0, \infty), f(t)=\exp (t)$, then, by performing the required calculations, we get

$$
0 \leq \frac{G^{2}(a, b)}{L(a, b)}-H(a, b) \leq \frac{1}{4} \frac{A(a, b)}{G^{2}(a, b)}(b-a)^{2}
$$

and

$$
0 \leq A(a, b)-\frac{G^{2}(a, b)}{L(a, b)} \leq \frac{1}{4} \frac{A(a, b)}{G^{2}(a, b)}(b-a)^{2}
$$


From the inequality (13) we also have

$$
\frac{G^{2}(a, b)}{L(a, b)} \leq \frac{1}{2}\left(A(a, b)+b^{\frac{a}{a+b}} a^{\frac{b}{a+b}}\right) .
$$

Acknowledgement. The author would like to thank the anonymous referees for valuable comments that have been implemented in the final version of the paper.

\section{References}

[1] Alomari M., Darus M. The Hadamard's inequality for s-convex function. Int. J. Math. Anal., 2008, vol. 2, no. 13-16, pp. 639-646.

[2] Alomari M., Darus M. Hadamard-type inequalities for s-convex functions. Int. Math. Forum, 2008, vol. 3, no. 37-40, pp. 1965-1975.

[3] Anastassiou G. A. Univariate Ostrowski inequalities, revisited. Monatsh. Math., 2002, vol. 135, no. 3, pp. 175-189.

[4] Anderson G. D., Vamanamurthy M. K., Vuorinen M. Generalized convexity and inequalities. J. Math. Anal. Appl., 2007, vol. 335, pp. 1294-1308.

[5] Barnett N. S., Cerone P., Dragomir S. S., Pinheiro M. R., Sofo A. Ostrowski type inequalities for functions whose modulus of the derivatives are convex and applications. Inequality Theory and Applications, vol. 2 (Chinju/Masan, 2001), 19-32, Nova Sci. Publ., Hauppauge, NY, 2003. Preprint: RGMIA Res. Rep. Coll., 2002, vol. 5, no. 2, art. 1 [Online http://rgmia.org/papers/v5n2/Paperwapp2q.pdf] .

[6] Beckenbach E. F. Convex functions. Bull. Amer. Math. Soc., 1948, vol. 54, pp. 439-460.

[7] Bombardelli M., Varošanec S. Properties of h-convex functions related to the Hermite-Hadamard-Fejér inequalities. Comput. Math. Appl., 2009, vol. 58, no. 9, pp. 1869-1877.

[8] Cristescu G. Hadamard type inequalities for convolution of h-convex functions. Ann. Tiberiu Popoviciu Semin. Funct. Equ. Approx. Convexity, 2010, vol. 8, pp. 3-11.

[9] Dragomir S. S. On the Ostrowski's inequality for mappings of bounded variation and applications. Math. Ineq. \& Appl., 2001, vol. 4, no. 1, pp. $33-40$.

[10] Dragomir S. S. An inequality improving the first Hermite-Hadamard inequality for convex functions defined on linear spaces and applications for semi-inner products. J. Inequal. Pure Appl. Math., 2002, vol. 3, no. 2, art. $31,8 \mathrm{p}$. 
[11] Dragomir S. S. An inequality improving the second Hermite-Hadamard inequality for convex functions defined on linear spaces and applications for semi-inner products. J. Inequal. Pure Appl. Math., 2002, vol. 3, no. 3, art. 35.

[12] Dragomir S. S. An Ostrowski like inequality for convex functions and applications. Revista Math. Complutense, 2003, vol. 16, no. 2, pp. 373-382.

[13] Dragomir S. S. Operator Inequalities of Ostrowski and Trapezoidal Type. Springer Briefs in Mathematics. Springer, New York, 2012. x+112 pp. ISBN: 978-1-4614-1778-1

[14] Dragomir S. S. Some new inequalities of Hermite-Hadamard type for GAconvex functions. Preprint RGMIA Res. Rep. Coll., 2015, vol. 18, art. 30. [http://rgmia.org/papers/v18/v18a30.pdf] .

[15] Dragomir S. S. Inequalities of Hermite-Hadamard type for HA-convex functions. Preprint RGMIA Res. Rep. Coll., 2015, vol. 18, art. 38. [http://rgmia.org/papers/v18/v18a38.pdf] .

[16] Dragomir S. S. New inequalities of Hermite-Hadamard type for HAconvex functions. Preprint RGMIA Res. Rep. Coll., 2015, vol. 18, art. 41. [http://rgmia.org/papers/v18/v18a41.pdf] .

[17] Dragomir S. S., Cerone P., Roumeliotis J., Wang S. A weighted version of Ostrowski inequality for mappings of Hölder type and applications in numerical analysis. Bull. Math. Soc. Sci. Math. Romanie, 1999, vol. 42(90), no. 4, pp. 301-314.

[18] Dragomir S. S., Fitzpatrick S. The Hadamard inequalities for s-convex functions in the second sense. Demonstratio Math., 1999, vol. 32, no. 4, pp. 687-696.

[19] Dragomir S. S., Fitzpatrick S. The Jensen inequality for s-Breckner convex functions in linear spaces. Demonstratio Math., 2000, vol. 33, no. 1, pp. 43-49.

[20] Dragomir S. S., Mond B. On Hadamard's inequality for a class of functions of Godunova and Levin. Indian J. Math., 1997, vol. 39, no. 1, pp. 1-9.

[21] Dragomir S. S., Pearce C. E. M. On Jensen's inequality for a class of functions of Godunova and Levin. Period. Math. Hungar., 1996, vol. 33, no. 2, pp. 93-100.

[22] Dragomir S. S., Pearce C. E. M. Quasi-convex functions and Hadamard's inequality. Bull. Austral. Math. Soc., 1998, vol. 57, pp. 377-385.

[23] Dragomir S. S., Pečarić J., Persson L.-E. Some inequalities of Hadamard type. Soochow J. Math., 1995, vol. 21, no. 3, pp. 335-341.

[24] Dragomir S. S., Rassias Th. M. (Eds) Ostrowski Type Inequalities and Applications in Numerical Integration. Kluwer Academic Publisher, 2002. 
[25] El Farissi A. Simple proof and refinement of Hermite-Hadamard inequality. J. Math. Ineq., 2010, vol. 4, no. 3, pp. 365-369.

[26] Işcan I. Hermite-Hadamard type inequalities for harmonically convex functions. Hacettepe Journal of Mathematics and Statistics, 2014, vol. 43, no. 6, pp. 935-942.

[27] Kikianty E., Dragomir S. S. Hermite-Hadamard's inequality and the pHH-norm on the Cartesian product of two copies of a normed space. Math. Inequal. Appl., 2010, vol. 13, no. 1, pp. 1-32.

[28] Pečarić J. E., Dragomir S. S. On an inequality of Godunova-Levin and some refinements of Jensen integral inequality. Itinerant Seminar on Functional Equations, Approximation and Convexity (Cluj-Napoca, 1989), pp. 263268, Preprint, 89-6, Univ. "Babeş-Bolyai", Cluj-Napoca, 1989.

Received April 19, 2017.

In revised form, July 11, $201 \%$.

Accepted July 11, $201 \%$.

Published online August 16, $201 \%$.

Mathematics, College of Engineering \& Science

Victoria University, PO Box 14428

Melbourne City, MC 8001, Australia;

DST-NRF Centre of Excellence in the Mathematical and Statistical Sciences,

School of Computer Science and Applied Mathematics

University of the Witwatersrand,

Private Bag 3, Johannesburg 2050, South Africa

E-mail: sever.dragomir@vu.edu.au 